\title{
THE ENUMERATION OF POINT LABELLED CHROMATIC GRAPHS AND TREES
}

\author{
T. L. AUSTIN
}

1. Introduction. Given $n$ points with $c_{1}$ of one colour, $c_{2}$ of another colour, up to $k$ colours, linear graphs are formed with the restriction that no line connects points of the same colour. Following fairly standard terminology, coloured graphs with this restriction will be called point chromatic graphs. Giving the points numerical labels running from 1 to $c_{i}$ for points of the $i$ th colour $(i=1,2, \ldots, k)$ forms point labelled chromatic graphs. Note that this description is slightly different from assigning a label and a colour independently to each point. If there are $N$ graphs of the first kind and $N^{*}$ of the second the relationship is

$$
\frac{n !}{c_{1} ! c_{2} ! \ldots c_{k} !} N=N^{*} \text {. }
$$

In this paper, relationships for enumerating these graphs are derived, along with simple expressions for the number of such trees (connected graphs with no cycles) and the number of such connected graphs of two colours with a single cycle.

2. Generating functions. Let $N\left(c_{1}, c_{2}, \ldots, c_{k} ; l\right)$ be the number of point labelled chromatic linear graphs with $l$ lines, $c_{1}$ labelled points of one colour, $c_{2}$ labelled points of another colour, etc., connected or in several parts, and let $C\left(c_{1}, c_{2}, \ldots, c_{k} ; l\right)$ be the number of such graphs that are connected. For convenience these will be written in vector notation, for instance, $N\left(c_{1}, c_{2}, \ldots, c_{k} ; l\right)=N(C, l)$ with $C=\left(c_{1}, c_{2}, \ldots, c_{k}\right)$.

It is clear that

$$
N(C, l)=\left(\frac{1}{2}\left(n^{2}-\sum_{i=1}^{k} c_{i}^{2}\right)\right), \quad n=c_{1}+c_{2}+\ldots+c_{k},
$$

since the fully connected labelled chromatic graph, that is, the graph with a line connecting each pair of differently coloured points has $c_{i}\left(n-c_{i}\right)$ lines at points of the $i$ th colour, hence has

$$
\begin{aligned}
& \frac{1}{2}\left(\left[n-c_{1}\right] c_{1}+\left[n-c_{2}\right] c_{2}+\ldots+\left[n-c_{k}\right] c_{k}\right) \\
= & \frac{1}{2}\left(n\left[c_{1}+c_{2}+\ldots+c_{k}\right]-\left[c_{1}^{2}+c_{2}^{2}+\ldots+c_{k}^{2}\right]\right) \\
= & \frac{1}{2}\left(n^{2}-\sum c_{i}^{2}\right)
\end{aligned}
$$

Received June 3, 1959. 
lines in all. $N(C, l)$ obviously arises by taking ordered selections of $l$ lines from this graph. hence (1) follows immediately.

Likewise

$$
N^{\prime}(C, l)=\left(\frac{1}{2}\left(n^{2}-\sum_{i=1}^{k} c_{i}^{2}\right)+l-1\right)
$$

with the prime indicating that lines in parallel are allowed follows in the same way, since this is just the form that (1) takes if the selection is done with replacement.

Introduce the exponential generating functions of the numbers $N(C, l)$ and $C(C, l)$ :

(2) $N\left(x_{1}, x_{2}, \ldots, x_{k} ; z\right)=N(X, z)$

$$
=\sum_{c_{1}=0}^{\infty} \sum_{c_{2}=0}^{\infty} \ldots \sum_{c_{k}=0}^{\infty} \sum_{l=0}^{\frac{1}{2}\left[n^{2}-\Sigma c_{i}^{2}\right]} \frac{x_{1}^{c_{1}}}{c_{1} !} \frac{x_{2}^{c_{2}}}{c_{2} !} \ldots \frac{x_{k}^{c_{k}}}{c_{k} !} z^{l} N(C, l)
$$

(3) $C\left(x_{1}, x_{2}, \ldots, x_{k} ; z\right)=C(X, z)$

$$
=\sum_{c_{1}=0}^{\infty} \sum_{c_{2}=0}^{\infty} \ldots \sum_{c_{k}=0}^{\infty} \sum_{l=0}^{\frac{1}{2}\left(n^{2}-\Sigma c_{i}^{2}\right)} \frac{x_{1}^{c_{1}}}{c_{1} !} \frac{x_{2}^{c_{2}}}{c_{2} !} \frac{x_{k}^{c_{k}}}{c_{k} !} z^{l} C(C, l)
$$

with $N(0, l)=C(0, l)=0$ by convention. Then the relation between the known $N$ and $C$ is given by

Theorem 1. $C(X, z)=\log [N(X, z)+1]$.

The proof follows from a slight modification of an argument by Gilbert (1). Thus represent by $N_{m}(C, l)$ the number of graphs which are in $m$ parts. A graph enumerated by $N_{m}(C, l)$ evidently consists of $m$ distinct connected graphs.

Thus, any point is in a connected graph with $i_{1}$ points of the first colour, $i_{2}$ points of the second colour, etc., and with $j$ lines, and the other $\left(c_{1}-i_{1}\right)$, $\left(c_{2}-i_{2}\right), \ldots,\left(c_{k}-i_{k}\right)$ points and $l-j$ lines are in a graph consisting of $m-1$ parts. The points can be labelled in

$$
\left(\begin{array}{l}
c_{1} \\
i_{1}
\end{array}\right)\left(\begin{array}{l}
c_{2} \\
i_{2}
\end{array}\right) \ldots\left(\begin{array}{c}
c_{k} \\
i_{k}
\end{array}\right)
$$

ways and therefore

(4) $N_{m}(C ; l)$

$$
\begin{array}{r}
=\sum_{i_{1}, i_{2}, \ldots, i_{k}, j} C\left(i_{1}, i_{2}, \ldots, i_{k} ; j\right) N_{m-1}\left(c_{1}-i_{1}, c_{2}-\right. \\
\left.\quad i_{2}, \ldots, c_{k}-i_{k} ; l-j\right) \\
\times\left(\begin{array}{c}
c_{1} \\
i_{1}
\end{array}\right)\left(\begin{array}{c}
c_{2} \\
i_{2}
\end{array}\right) \ldots\left(\begin{array}{c}
c_{k} \\
i_{k}
\end{array}\right) .
\end{array}
$$

Next, choosing one of the points in the graph in $m-1$ parts and repeating 
the argument, it follows that (with superscripts to distinguish the connected graphs)

(5) $\quad N_{m}(C, l)$

$$
\begin{aligned}
=\frac{1}{2}{ }_{I^{(1)}, I^{(2)}, j^{(1)}, j^{(2)}} C\left(i_{1}^{(1)}, i_{1}^{(1)}, \ldots, i_{k}^{(1)} ; j^{(1)}\right) C\left(i_{1}^{(2)}, i_{1}^{(2)}, \ldots, i_{k}^{(2)} ; j^{(2)}\right) \\
\quad \times N_{m-2}\left(c_{1}-i_{1}^{(1)}-i_{2}^{(2)}, \ldots, c_{k}-i_{k}^{(1)}-i_{k}^{(2)} ; l-j^{(1)}-j^{(2)}\right) \\
\quad \times \frac{c_{1} !}{i_{1}^{(1)} ! i_{1}^{(2)} !\left(c_{1}-i_{1}^{(1)}-i_{2}^{(2)}\right) !} \cdots \frac{c_{k} !}{i_{k}^{(1)} ! i_{k}^{(2)} !\left(c_{k}-i_{k}^{(1)}-i_{k}^{(2)}\right) !}
\end{aligned}
$$

with the factor $\frac{1}{2}$ entering to account for order between the two distinct connected parts. Continuing this process, one obtains

(6) $\quad N_{m}(C, l)=\frac{1}{m !} \sum_{I^{(1)}} \sum_{\ldots, I^{(m)}} C\left(I^{(1)} ; j^{(1)}\right) C\left(I^{(2)} ; j^{(2)}\right) \ldots C\left(I^{(m)} ; j^{(m)}\right)$

$$
\times \frac{c_{1} !}{i_{1}^{(1)} ! i_{1}^{(2)} ! \ldots i_{1}^{(m)} !} \frac{c_{2} !}{i_{2}^{(1)} ! i_{2}^{(2)} ! \ldots i_{2}^{(m)} !} \cdots \frac{c_{k} !}{i_{k}^{(\overline{1}) !} ! i_{k}^{(2)} ! \ldots i_{k}^{(\bar{m})} !}
$$

Multiplying both sides of (6) by

$$
\frac{x_{1}^{c_{1}}}{c_{1} !} \cdot \frac{x_{2}^{c_{2}}}{c_{2} !} \ldots \frac{x_{k}^{c_{k}}}{c_{k} !} z^{l}
$$

and summing in turn over $c_{1}, c_{2}, \ldots, c_{k}$ and $l$ gives

$$
\text { (7) } \begin{aligned}
\sum_{c_{1}=0}^{\infty} \sum_{c_{2}=0}^{\infty} \ldots \sum_{c_{k}=0}^{\infty} \sum_{l=0}^{\frac{1}{2}\left[n^{2}-\Sigma c_{i}^{2}\right]} \frac{x_{1}^{c_{1}}}{c_{1} !} \frac{x_{2}^{c_{2}}}{c_{2} !} \ldots \frac{x_{k}^{c_{k}}}{c_{k} !} z^{l} N_{m}(C ; l) \\
=N_{m}\left(x_{1}, x_{2}, \ldots, x_{k} ; z\right)=N_{m}(X ; z)=\frac{1}{m !} C^{m}(X, z) .
\end{aligned}
$$

Here $N_{0}=C^{0}=0$. Then since

$$
N(X ; z)=\sum_{m=1}^{\infty} N_{m}(X, z),
$$

and by (7), the thereom follows immediately.

3. Trees. A tree is a connected linear graph with no cycles (closed path of points). Obviously this is the special case $C(C, n-1)$.

Theorem II. The number of point labelled chromatic trees, $C(C, n-1)$, on $n$ points with $c_{1}$ labelled points of one colour, $c_{2}$ labelled points of another colour, with $k$ colours in all, is

$$
C(C, n-1)=n^{k-2}\left(n-c_{1}\right)^{c_{1}-1}\left(n-c_{2}\right)^{c_{2}-1} \ldots\left(n-c_{k}\right)^{c_{k-1}} .
$$

Form the fully connected point labelled point chromatic graph on $n=c_{1}+c_{2}+\ldots+c_{k}$ points and consider the matrix $A$, with

$$
\begin{aligned}
& a_{i i}=(\text { the number of lines at point } i) i=1,2, \ldots, n \\
& a_{i j}=-(\text { the number of lines between points } i \text { and } j), i \neq j .
\end{aligned}
$$


Since all points are distinguishable either by label or colour, there is no ambiguity in the above definition and in fact

$$
\begin{aligned}
& a_{i i}=\left(n-c_{i}^{\prime}\right) \\
& a_{i \jmath}=\left\{\begin{array}{r}
-1 \text { if points } i \text { and } j \text { are of different colour } \\
0 \text { otherwise. }
\end{array}\right.
\end{aligned}
$$

Where $c_{i}{ }^{\prime}$ is the number of points of the same colour as point $i$.

For concreteness, this matrix with $c_{1}=1, c_{2}=2, c_{3}=3, n=6$ is

$$
\left(\begin{array}{rrrrrr}
5 & -1 & -1 & -1 & -1 & -1 \\
-1 & 4 & 0 & -1 & -1 & -1 \\
-1 & 0 & 4 & -1 & -1 & -1 \\
-1 & -1 & -1 & 3 & 0 & 0 \\
-1 & -1 & -1 & 0 & 3 & 0 \\
-1 & -1 & -1 & 0 & 0 & 3
\end{array}\right)
$$

Since all rows and columns of $A$ sum to zero, all $(n-1) \times(n-1)$ cofactors are equal. Trent (4) has shown that this cofactor is the number of trees in a fully connected graph which has the incidence matrix $B$, with

$$
\begin{aligned}
& b_{i i}=0 \\
& b_{i j}=-a_{i j}, \quad i \neq j .
\end{aligned}
$$

Thus to enumerate trees, it is necessary to evaluate a determinant of the form

$$
\left|\begin{array}{ccccr}
\gamma_{1} I\left(d_{1}\right) & -V_{12} & -V_{13} & \ldots & -V_{1 k} \\
-V_{21} & \gamma_{2} I\left(d_{2}\right) & \cdots & & \vdots \\
\vdots & & & & \gamma_{k} I\left(d_{k}\right)
\end{array}\right|=\operatorname{Det} \Gamma
$$

with $V_{i j}\left(d_{i} x d_{j}\right)=\left\{v_{i j}\right\} ; v_{i j}=1$ all $i, j$, the $\gamma$ 's scalars, and $I\left(d_{i}\right)$ the identity matrix of dimension $d_{i}$.

Since $\Gamma$ is symmetric, it is necessary only to consider its latent roots to evaluate its determinant. Hence consider the system of equations

$$
\Gamma\left(\begin{array}{c}
Y_{1} \\
Y_{2} \\
\cdot \\
\cdot \\
Y_{k}
\end{array}\right)=\lambda\left(\begin{array}{c}
Y_{1} \\
Y_{2} \\
\cdot \\
\cdot \\
Y_{k}
\end{array}\right)
$$

with $Y_{i}=Y_{i}\left(d_{i} x 1\right)=\left(y_{i 1}, y_{i 2}, \ldots, y_{i d i}\right)^{\prime}$.

If $\lambda$ is different from each of $\gamma_{1}, \gamma_{2}, \ldots, \gamma_{k}$ then a consideration of the equations two at a time shows that each vector $Y_{i}$ must be a constant vector. Thus (8) can be written as 


$$
\sum_{j=1}^{k}\left[\left(\gamma_{j}+d_{j}\right) \delta_{i j}-d_{j}\right] Y_{j}=\lambda_{i} Y_{i} \quad(i=1, \ldots, k)
$$

which contains exactly $k$ independent equations. This accounts for $k$ latent roots, each different from $\gamma_{1}, \gamma_{2}, \ldots, \gamma_{k}$ and the product of these $k$ roots is the determinant $D=\left|\left(\gamma_{j}+d_{j}\right) \delta_{i k}-d_{k}\right|$.

Next if $\lambda=\gamma_{1}$ (say), then clearly $Y_{2}=Y_{3}=\ldots=Y_{k}=0, \sum_{i} y_{l i}=0$, the root $\gamma_{1}$ occurs $d_{1}-1$ times, and similarly for $\lambda=\gamma_{2}, \lambda=\gamma_{3}$, etc. Therefore it follows that

$$
\text { Det } \Gamma=\gamma_{1}^{d_{1}-1} \gamma_{2}^{\boldsymbol{d}_{2}-1} \ldots \gamma_{k}^{d_{k-1}} D \text {. }
$$

Substituting in $(10)$ the values for an $(n-1) \times(n-1)$ cofactor of $A$,

$$
\begin{aligned}
& \gamma_{1}=\left(n-c_{1}\right), \gamma_{2}=\left(n-c_{2}\right), \ldots, \gamma_{k}=\left(n-c_{k}\right) ; \\
& d_{1}=c_{1}-1, d_{2}=c_{2}, d_{3}=c_{3}, \ldots, d_{k}=c_{k}
\end{aligned}
$$

(10) becomes

$$
\left(n-c_{1}\right)^{c_{1}-2}\left(n-c_{2}\right)^{c_{2}-1} \ldots\left(n-c_{k}\right)^{c_{k}-1}\left|\begin{array}{c}
\left(n-c_{1}\right)-c_{2}-c_{3} \ldots-c_{k} \\
\left(1-c_{1}\right)\left(n-c_{2}\right)-c_{3} \ldots-c_{k} \\
\vdots \\
\left(1-c_{1}\right)-c_{2}-c_{3} \ldots\left(n-c_{k}\right)
\end{array}\right|
$$

The determinant can be evaluated simply by writing it as

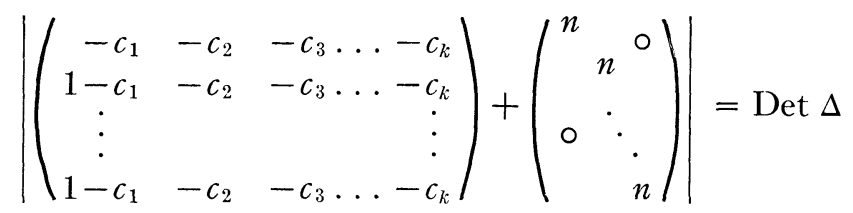

which can be expanded about the terms of the diagonal matrix, that is,

$$
\text { Det } \begin{aligned}
\Delta & =n^{k}-n^{k-1}\left(c_{1}+c_{2}+\ldots+c_{k}\right) \\
& +n^{k-2}\left[c_{1} c_{2}+c_{2}\left(1-c_{1}\right)+c_{1} c_{3}+c_{3}\left(1-c_{3}\right)+\ldots\right. \\
& \left.\ldots+c_{1} c_{k}+c_{k}\left(1-c_{1}\right)\right]
\end{aligned}
$$

which are all the terms since the first matrix is of rank two. Using the relation $c_{1}+c_{2}+\ldots+c_{k}=n$, (13) becomes

$$
\text { Det } \Delta=n^{k-2}\left[c_{2}+c_{3}+\ldots+c_{k}\right]=n^{k-2}\left(n-c_{1}\right) \text {. }
$$

Putting (11) and (14) together the theorem follows.

4. Connected graphs with one cycle. After trees the next graph in order of complexity is a graph with one cycle. This section derives the number of point labelled chromatic graphs with two colours and one cycle. For convenience, the notation of this section is different from that of the previous sections. Note that lines in parallel (cycles of length 2) are allowed. 
Before stating the main result of this section it will be necessary to establish a few preliminaries.

A rooted tree is a tree in which one point, the root, has been preferred. In a labelled tree on $n$ points, any point may be preferred and all points are distinguishable, hence there are $n$ rooted trees for each root free tree.

Now let $R_{p q}$ be the number of rooted chromatic trees, with $p$ labelled black points and $q$ labelled white points, and let $T_{p q}$ be the number of such root free trees. Then since all points are distinguishable and since each point may be preferred in turn as the root, it follows that

$$
\begin{aligned}
R_{p q} & =(p+q) T_{p q} \\
& =p T_{p q}+q T_{p q} \\
& =r_{p q}+r_{p q}{ }^{\prime}
\end{aligned}
$$

with $r_{p q}$ the number of trees with a black root and $r_{p q}{ }^{\prime}$ the number with a white root.

Introduce the counting series,

$$
\begin{aligned}
R(x, y) & =\sum_{p} \sum_{q} \frac{x^{p}}{p !} \frac{y^{q}}{q !} R_{p q} \\
T(x, y) & =\sum_{p} \sum_{q} \frac{x^{p}}{p !} \frac{y^{q}}{q !} T_{p q} \\
r(x, y) & =\sum_{p} \sum_{q} \frac{x^{p}}{p !} \frac{y^{q}}{q !} r_{p q} \\
r^{\prime}(x, y) & =\sum_{p} \sum_{q} \frac{x^{p}}{p !} \frac{y^{q}}{q !} r_{p q}^{\prime}
\end{aligned}
$$

and let $r_{p q}(n)$ be the number of trees with a black root and with exactly $n$ lines at the root. Then a slight modification of an argument by Polya (2) also given in Riordan (3, page 127) yields a relationship between $r(x, y)$ and $r^{\prime}(x, y)$. Thus

$$
r_{p q}(1)=p r_{p-1, q}^{\prime}
$$

because there are $p$ labels for the root and the single line connects the root to a white point which may be regarded as the root of a tree with $p-1$ black and $q$ white points.

Similarly,

$$
\begin{aligned}
r_{p q}(2) & =\frac{1}{2 !} p \sum_{i=1}^{p-1} \sum_{j=1}^{q-1}\left(\begin{array}{c}
p-1 \\
i
\end{array}\right)\left(\begin{array}{l}
q \\
j
\end{array}\right) r_{p-1-i, q-j}^{\prime} r_{i j}^{\prime} \\
& =\frac{1}{2} p \sum_{i=1}^{p-1}\left(\begin{array}{c}
p-1 \\
i
\end{array}\right)\left[r_{p-1-i,}^{\prime}+r_{i}^{\prime}\right]^{Q}
\end{aligned}
$$

symbolically, with $\left[r_{i},\right]^{j}=r_{i j}$ and $r_{n 0}{ }^{\prime}=r_{0 n}=0$, because the labels for the 
two (white rooted) trees added to the two lines at the (black) root may be chosen in

$$
\left(\begin{array}{c}
p-1 \\
i
\end{array}\right)\left(\begin{array}{l}
q \\
j
\end{array}\right)
$$

ways when one of the trees has $i$ black and $j$ white points. Because of the labels, the added trees are not alike. The factor (1/2!) accounts for order between the two lines. In general, it is clear that

$$
r_{p q}(n)=\frac{p}{n !} \sum\left(\begin{array}{c}
p-1 \\
i_{1}, i_{2}, \ldots, i_{n-1}
\end{array}\right)\left[r_{i_{1}}^{\prime},+r_{i_{2}}^{\prime},+\ldots+r_{p-1-i 1-\ldots-i n-1}^{\prime}\right]^{q}
$$

since the number of ways of assigning labels to trees with $i_{1}, i_{2}, \ldots$ black and $j_{1}, j_{2}, \ldots$ white points is

$$
\left(\begin{array}{c}
p-1 \\
i_{1}, i_{2}, \ldots, i_{n-1}
\end{array}\right)\left(\begin{array}{c}
q \\
j_{1}, j_{2}, \ldots, j_{n-1}
\end{array}\right)
$$

From $r_{10}=1$ and since obviously

$$
r_{p q}=\sum_{n=1} r_{p q}(n)
$$

it follows from (22) at once that

$$
r(x, y)=x \exp r^{\prime}(x, y)
$$

and by an exactly similar argument,

$$
r^{\prime}(x, y)=y \exp r(x, y) .
$$

From (23) and (24),

$$
r(x, y)=x \exp [y \exp r(x, y)] \text { results. }
$$

Recalling the Lagrange formula,

$$
\begin{aligned}
t & =x \phi(t) \\
f(t) & =f(0)+\sum_{n=1}^{\infty} \frac{x^{n}}{n !} \frac{d^{n-1}}{d t^{n-1}}\left[f^{\prime}(t) \phi^{n}(t)\right]_{t=0},
\end{aligned}
$$

and putting $f(t)=t, t=r(x, y)$,

$$
\phi(t)=\phi(r[x, y])=\exp [y \exp r(x, y)],
$$

it follows that

$$
r(x, y)=\sum_{p} \sum_{q} \frac{x^{p}}{p !} \frac{y^{q}}{q !} p^{q} q^{p-1}
$$

and similarly,

$$
r^{\prime}(x, y)=\sum_{p} \sum_{q} \frac{x^{p}}{p !} \frac{y^{q}}{q !} p^{q-1} q^{p} .
$$


From (26), (27), and (15),

$$
T(x, y)=\sum_{p} \sum_{q} \frac{x^{p}}{p !} \frac{y^{q}}{q !} p^{q-1} q^{p-1}
$$

follows. This is the result (for the case of two colours) of Theorem II.

The result may now be stated.

THEOREM III. The number of connected point labelled chromatic graphs with p labelled black points and $q$ labelled white points with one cycle of length $2 m$ is

$$
\frac{1}{2}(p+q-m)(p)_{m}(q)_{m} p^{q-m-1} q^{p-m-1} \text { for } m>1
$$

and

$$
(p+q-1) p^{q-1} q^{p-1} \text { for } m=1 .
$$

To enumerate (two coloured) labelled chromatic graphs with one cycle. note that the cycle must be even, with the same number of white as black points because of the chromatic condition. The essential enumeration is therefore of such graphs with a single cycle of length $2 \mathrm{~m}$. These graphs may be enumerated by a theorem due to Polya $(2 ; 3)$, since they may be regarded as being formed by placing white rooted trees (say) at the vertices of an $m$-sided polygon and then replacing the lines of the polygon by roots of black rooted trees to form a polygon of $2 m$ vertices. Every permutation of labels for the black roots clearly gives rise to a different polygon of $2 m$ points while, on the other hand, any permutation of labels for the white roots which is a reflection or rotation of the original $m$-sided polygon is equivalent to some permutation of the black labels. Thus the different cycles formed are the result of a direct product of two groups, the identity group and the dihedral group.

Letting

$$
d_{2 m}\left(u_{1}, u_{2}, v_{1}, v_{2}\right)=\sum_{\alpha, \beta, \gamma, \delta} u_{1}^{\alpha} u_{2}^{\beta} \frac{v_{1}^{\gamma}}{\gamma !} \frac{v_{2}^{\delta}}{\delta !} d_{\alpha \beta \gamma \delta}(2 m)
$$

be the enumerator of such graphs by number of points and point labels, then by the theorem and from a simple extension of a problem in Riordan (3, p. 158),

$$
\begin{aligned}
& d_{2 m}\left(u_{1}, u_{2}, v_{1}, v_{2}\right)=I_{m}\left(r\left[u_{1}, u_{2}, v_{1}, v_{2}\right]\right) D_{m}\left(r^{\prime}\left[u_{1}, u_{2}, v_{1}, v_{2}\right],\right. \\
& \left.r^{\prime}\left[u_{1}^{2}, u_{2}^{2}\right], \ldots, r^{\prime}\left[u_{1}^{m}, u_{2}^{m}\right]\right), \quad \text { for } m>2 ; \\
& d_{2 m}\left(u_{1}, u_{2}, v_{1}, v_{2}\right)=S_{m}\left(r\left[u_{1}, u_{2}, v_{1}, v_{2}\right], r\left[u_{1}^{2}, u_{2}^{2}\right], \ldots, r\left[u_{1}^{m}, u_{2}^{m}\right]\right) \\
& S_{m}\left(r^{\prime}\left[u_{1}, u_{2}, v_{1}, v_{2}\right], r^{\prime}\left[u_{1}^{2}, u_{2}^{2}\right], \ldots, r^{\prime}\left[u_{1}^{m}, u_{2}^{m}\right]\right) \quad \text { for } m=1,2 \text {, }
\end{aligned}
$$

with $r\left(u_{1}, u_{2}, v_{1}, v_{2}\right)$ and $r^{\prime}\left(u_{1}, u_{2}, v_{1}, v_{2}\right)$ the enumerators of (black and white) rooted trees by number of points and number of point labels,

$$
r\left(u_{1}, u_{2}\right) \equiv r\left(u_{1}, u_{2}, 0,0\right)
$$


$I_{m}(t)=t^{m}$, the cycle index of the identity group and $D_{m}\left(t_{1}, t_{2}, \ldots, t_{m}\right)$ the cycle index of the dihedral group,

$$
\begin{aligned}
D_{m}\left(t_{1}, t_{2}, \ldots, t_{m}\right) & =\frac{1}{2 m} \sum_{i \mid m} \phi(i) t_{i}^{m / i}+\frac{1}{2} t_{1} t_{2}^{(m-1) / 2}, & & \text { for } m \text { odd } ; \\
& =\frac{1}{2 m} \sum_{i \mid m} \phi(i) t_{i}^{m / i}+\frac{1}{4} t_{2}^{m / 2-1}\left(t_{1}^{2}+t_{2}\right), & & \text { for } m \text { even. }
\end{aligned}
$$

Here $\phi(i)$ is the Euler totient function, the number of integers not greater than $i$ and relatively prime to $i$,

$$
S_{m}\left(t_{1}, t_{2}, \ldots, t_{m}\right)=\frac{1}{m !} \sum \frac{m !}{j_{1} ! 1^{j_{1}} j_{2} ! 2^{j_{2}} \ldots j_{m} ! m^{j_{m}}} t_{1}^{j_{1}} t_{2}^{j_{2}} \ldots t_{m}^{j_{m}}
$$

is the cycle index of the symmetric group, the sum extending over all solutions of

$$
j_{1}+2 j_{2}+\ldots+m j_{m}=m .
$$

The substitutions $x=u_{1} v_{1}, y=u_{2} v_{2}$ in the definition of $d_{2 m}\left(u_{1}, u_{2}, v_{1}, v_{2}\right)$ change it to the form

$$
d_{2 m}\left(u_{1}, u_{2}, x, y\right)=\sum_{i, \jmath} u_{1}^{i} u_{2}^{j} \sum d_{\gamma+i, \delta+j, \gamma, \delta} \frac{x^{\gamma}}{\gamma !} \frac{y^{\delta}}{\delta !} .
$$

Therefore, the numbers required, $d_{\gamma \delta \gamma \delta}$ are enumerated by

$$
d_{2 m, 0}(x, y)=\sum d_{\gamma \delta \gamma \delta} \frac{x^{\gamma}}{\gamma !} \frac{y^{\delta}}{\delta !}
$$

which from (29) is

$$
\begin{aligned}
d_{2 m, 0}(x, y) & =\frac{1}{2 m} r^{m}(x, y) r^{\prime m}(x, y) & & \text { for } m>1 \\
& =r(x, y) r^{\prime}(x, y) & & \text { for } m=1 .
\end{aligned}
$$

Recalling

$$
r(x, y)=x \exp [y \exp r(x, y)]
$$

and using the Lagrange formula again with $f(t)=t^{m}$, one obtains

$$
r^{m}(x, y)=\sum_{p} \sum_{q} \frac{x^{p}}{p !} \frac{y^{q}}{q !}(p)_{m} p^{q-1} q^{p-m} m
$$

Also from (24)

$$
\begin{aligned}
r^{m}(x, y) r^{\prime m}(x, y) & =r^{m}(x, y) y^{m} \exp m r(x, y) \\
& =r^{m}(x, y) y^{m} \sum_{n=0}^{\infty} \frac{m^{n} r^{n}(x, y)}{n !} \\
r^{m}(x, y) r^{\prime m}(x, y) & =y^{m} \sum m^{n-m} \frac{r^{n}(x, y)}{n !}(n)_{m}
\end{aligned}
$$

and by (30) 


$$
\begin{aligned}
{\left[r(x, y) r^{\prime}(x, y)\right]^{m} } & =y^{m} \sum_{p} \sum_{q} \frac{x^{p}}{p !} \frac{y^{q}}{q !} p^{q-1} \sum_{n}(n)_{m} n\left(\begin{array}{l}
p \\
n
\end{array}\right) m^{n-m} q^{p-n} \\
& =y^{m} \sum_{p} \sum_{q} \frac{x^{p}}{p !} \frac{y^{q}}{q !} p^{q-1}(p)_{m} \sum_{n}\left(\begin{array}{l}
p-m \\
n-m
\end{array}\right) n m^{n-m} q^{p-n} .
\end{aligned}
$$

Setting $n=n-m+m$, we have

$$
\begin{aligned}
\sum\left(\begin{array}{l}
p-m \\
n-m
\end{array}\right) n m^{n-m} q^{p-n} & \\
& =\sum_{n}\left(\begin{array}{l}
p-m \\
n-m
\end{array}\right)(n-m) q^{p-n} m^{n-m}+m \sum_{n}\left(\begin{array}{l}
p-m \\
n-m
\end{array}\right) q^{p-n} m^{n-m} \\
& =(p-m) \sum_{n}\left(\begin{array}{l}
p-m-1 \\
n-m-1
\end{array}\right) q^{p-n} m^{n-m}+m \sum_{n}\left(\begin{array}{l}
p-m \\
n-m
\end{array}\right) q^{p-n} m^{n-m} \\
& =(p-m) m(q+m)^{p-m-1}+m(q+m)^{p-m} \\
& =m(q+m)^{p-m-1}(q+p)
\end{aligned}
$$

and therefore

$$
\begin{aligned}
{\left[r(x, y) r^{\prime}(x, y)\right]^{m} } & =y^{m} \sum_{p} \sum_{q} \frac{x^{p}}{p !} \frac{y^{q}}{q !} p^{q-1}(p)_{m} m(q+m)^{p-m-1}(p+q) \\
& =\sum_{p} \sum_{q} \frac{x^{p}}{p !} \frac{y^{q}}{q !}(p+q-m)(p)_{m}(q)_{m} p^{q-m-1} q^{p-m-1} .
\end{aligned}
$$

From (31) it follows at once that

$$
\begin{aligned}
d_{2 m, 0}(x, y) & =\frac{1}{2} \sum_{p} \sum_{q} \frac{x^{p}}{p !} \frac{y^{q}}{q !}(p+q-m)(p)_{m}(q)_{m} p^{q-m-1} q^{p-m-1} & \\
& =\sum \sum \frac{x^{p}}{p !} \frac{y^{q}}{q !}(p+q-1) p^{q-1} q^{p-1} & \text { for } m>1
\end{aligned}
$$

5. Remarks. Consider a fully point labelled but otherwise unrestricted tree on $n$ points (with no chromatic conditions). Construct from it a point chromatic tree as follows. Select any point and give to it one of $k$ colours. Give each point which shares a line with the first point one of some other $k-1$ colours arbitrarily, and continue until all points of the tree are coloured. No two points which share a line can have the same colour from this procedure and so the result is a point labelled point chromatic tree with the numerical labels running from 1 to $n$. On the first step, there are $k$ choices of colours and $k-1$ choices on each of the succeeding steps, hence a total of $k(k-1)^{n-1}$ choices in all. From a result of Cayley's, it is known that the number of point labelled trees is $n^{n-2}$. Therefore the total number of distinguishable point chromatic point labelled trees that can be formed from the above procedure is $k(k-1)^{n-1} n^{n-2}$. 
Next if the trees enumerated by $C(C, n-1)$ of Theorem II are re-labelled by running the numerical labels from 1 to $n$ and assigning them to the points independently of the point colours, there are

$$
\frac{n !}{c_{1} ! c_{2} ! \ldots c_{k} !}
$$

such assignments and so it follows at once that

$$
\sum \frac{n !}{c_{1} ! c_{2} ! \ldots c_{k} !} C\left(c_{1}, c_{2}, \ldots, c_{k} ; n-1\right)=k(k-1)^{n-1} n^{n-2}
$$

with the sum running over all partitions of $n$ into $k$ parts. Equation (33) provides a useful check on the enumeration and leads to the interesting identity

$$
\begin{aligned}
\sum \frac{n !}{c_{1} ! c_{2} ! \ldots c_{k} !}\left(n-c_{1}\right)^{c_{1}-1}\left(n-c_{2}\right)^{c_{2}-1} \ldots\left(n-c_{k}\right)^{c_{k}-1} & \\
= & =k(k-1)^{n-1} n^{n-2}
\end{aligned}
$$

or, for the more manageable case $k=2$,

$$
\sum_{i=1}^{n-1}\left(\begin{array}{c}
n \\
i
\end{array}\right) i^{n-i-1}(n-i)^{i-1}=2 n^{n-2}
$$

\section{REFERENCES}

1. E. N. Gilbert, Enumeration of labelled graphs, Can. J. Math., 8 (1956), 405-411.

2. G. Polya, Kombinatorische Anzahlbestimmungen für Gruppen, Graphen, und chemische Verbindungen, Acta. Math., 68 (1937).

3. John Riordan, An introduction to combinatorial analysis (New York, 1958).

4. H. M. Trent, $A$ note on the enumeration and listing of all possible trees in a connected linear graph, Proc. Nat. Acad. Sci., 40 (1954), 1004-1007.

Technical Operations, Inc.

Fort Monroe, Virginia 